\title{
Pseudo-Leptomeningeal Contrast Enhancement at 3T in Pediatric Patients Sedated by Propofol
}

\author{
(D)A.M. McKinney, (D)A. Chacko Achanaril, DB. Knoll, DD.R. Nascene, and (D) R.S. Gawande
}

\begin{abstract}
BACKGROUND AND PURPOSE: Propofol is a cerebral vasoconstrictor that modulates cerebral perfusion by decreasing the metabolic rate of oxygen. Because younger children often undergo intravenous sedation for MR imaging, this study set out to evaluate the degree of leptomeningeal contrast enhancement on $3 T$ postcontrast brain MR imaging and to determine whether this phenomenon relates to sequence, sedation dosage, or patient age or weight.
\end{abstract}

MATERIALS AND METHODS: During a 2-year period, of 152 children 1-5 years of age who underwent MR imaging, 43 were included for MRI review. Of these, 37 underwent postcontrast imaging with either solely gradient-echo TIWI $(n=20)$ or spin-echo TIWI ( $n=17)$; notably, 6 patients underwent both sequences. Three neuroradiologists separately graded the degree of leptomeningeal contrast enhancement (grades $0-3$ ) that was correlated with various factors and calculated the interobserver reliability.

RESULTS: For the 43 patients, the mean patient age was $3.1 \pm 1.4$ years. The leptomeningeal contrast-enhancement grade was significantly greater $(P<.0001)$ on spin-echo TIWI (1.9-2.1) versus gradient-echo TIWI (1.2-1.4). Patient weight $(r=-0.366$ to $-.418, P=.003-.01)$ and age $(r=-0.315$ to $-0.418, P=.004-.032)$ moderately and inversely correlated with the leptomeningeal contrast-enhancement grade, while the propofol dosage, sedation duration, and time to TTWI post-contrast administration did not (each, $P>.05$ ). The interobserver $\kappa$ was strong regarding the leptomeningeal contrast-enhancement grade on both spin-echo T1WI $(\kappa=0.609-0.693, P<.0001)$ and gradientecho TIWI $(\kappa=0.567-0.698, P<.0001)$.

CONCLUSIONS: Leptomeningeal contrast enhancement (or "pseudo"-leptomeningeal contrast enhancement) occurs with a greater frequency and degree on 3T postcontrast spin-echo TIWI relative to gradient-echo TIWI in younger children sedated with propofol and should not be mistaken for disease. This phenomenon may be more prominent with lower age or size and may arise from propofol-induced vascular smooth-muscle dilation.

ABBREVIATIONS: GE = gradient-echo; $\mathrm{LMCE}=$ leptomeningeal contrast enhancement; $\mathrm{SE}=$ spin-echo; $\mathrm{TTI}=$ time to postcontrast Tl-weighted imaging

$\mathbf{E}^{\mathrm{x}}$ xtra-axial enhancement in the CNS can be either leptomeningeal, occurring along the surface of the brain and subarachnoid space, or pachymeningeal, comprising the dura and its reflections. ${ }^{1}$ The vessels within the pachymeninges do not have a blood-brain barrier, which causes the typical appearance of thin, linear, and smooth enhancement on postcontrast T1WI following the intravenous administration of gadolinium-based contrast agents; in contrast, the main mechanism of leptomeningeal con-

Received February 13, 2018; accepted after revision June 5.

From the Department of Radiology (A.M.M., A.C.A., D.R.N.), Neuroradiology Division, University of Minnesota, Minneapolis, Minnesota; Department of Radiology (B.K.), Hennepin County Medical Center, Minneapolis, Minnesota; and Department of Radiology (R.S.G.), Johns Hopkins University, Baltimore, Maryland.

Please address correspondence to Alexander M. McKinney, MD, University of Minnesota, Department of Radiology, 420 Delaware St SE, MMC 292, Minneapolis, MN 55455; e-mail: mckinrad@umn.edu

http://dx.doi.org/10.3174/ajnr.A5736 trast enhancement (LMCE) (ie, pial enhancement) is disruption of the blood-brain barrier. ${ }^{1,2}$

Often, children younger than 8 years of age require sedation to undergo a high-quality MR imaging examination. Anesthetic agents have been shown to cause changes in cerebral homeostasis and vascular reactivity. ${ }^{3-5}$ Such agents can cause a global decrease in cerebral metabolism, with resultant decreases in both CBF and CBV. ${ }^{3-5}$ Prior studies have also demonstrated that 2,6 diisopropyl phenol (propofol) can modulate CBF by decreasing the metabolic rate of oxygen; in addition, speculation based on animal studies suggests that propofol can dilate vascular smooth muscle in other regions of the body. ${ }^{6}$

The basis of this study is that the authors had noted prominent LMCE on brain MR imaging in some sedated children, but based on clinical notes, they neither were acutely ill nor exhibited meningeal signs. Thus, this study was initiated to determine whether 


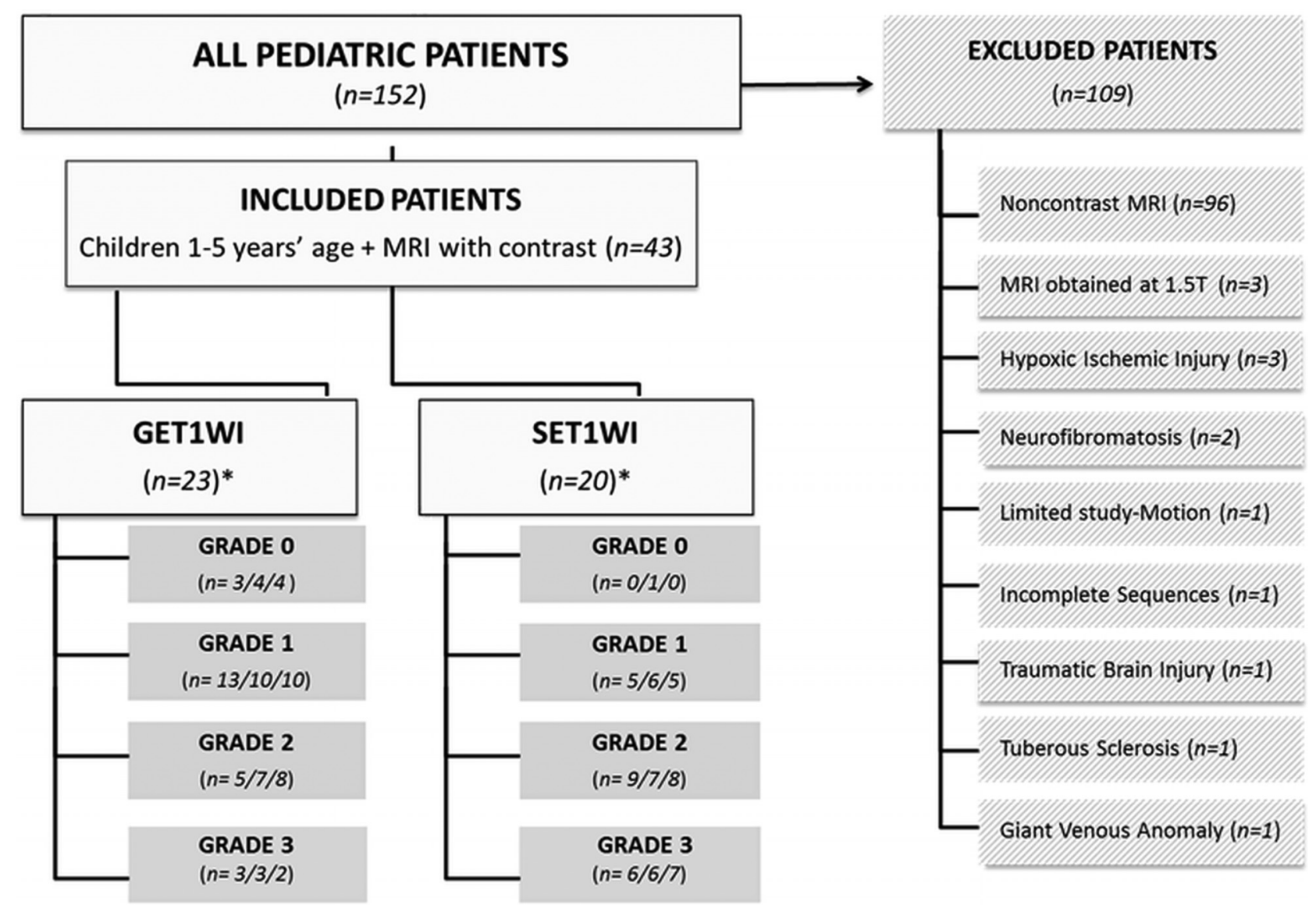

*Data of 3 reviewers; Note: $n=6$ underwent both GET1WI and SET1WI

FIG 1. Organization chart showing the makeup of 43 children included in this study and grades per reviewer.

the degree of this phenomenon of apparent LMCE (termed here "pseudo"-LMCE) relates to the type of T1WI sequence, time to acquiring postcontrast T1WI, propofol dosage, or various patient demographics.

\section{MATERIALS AND METHODS}

This retrospective study was performed after Hennepin County Medical Center, Minneapolis, Minnesota, review board approval. Review of the imaging data base and electronic clinical records yielded 152 healthy pediatric patients between the ages of 1 and 5 years who underwent $3 \mathrm{~T}$ brain MR imaging and were sedated with intravenous propofol between November 2011 and November 2013. Inclusion criteria were the following: 1) ages were between 1 and 5 years; 2) either axial gradient-echo (GE) T1WI or spin-echo (SE) T1WI was performed; 3) the child received gadoliniumbased intravenous contrast; 4) intravenous propofol was used for sedation; 5) the patient had either normal examination findings or only mild, nonacute, and noncongenital abnormalities (eg, $<5$ white matter foci); and 6) the patient had not had meningitis or other clinical diseases that could cause LMCE. Exclusion criteria consisted of the following: studies performed on a $1.5 \mathrm{~T}$ magnet, an incomplete MR imaging examination, moderate-to-severe structural abnormalities, or clinical signs of meningitis (Fig 1). Anesthesia was induced via intravenous administration of propofol by a pediatric intensivist, without the use of inhalational anesthetics, akin to sedation methods described previously. ${ }^{7}$

\section{MR Imaging Technique}

All studies were performed on a single 3T MR unit (Intera; Philips Healthcare, Best, the Netherlands), with sedation performed by a pediatric intensivist. The imaging parameters for GE TIWI were a volumetric acquisition of $9.8 \mathrm{~ms} / 4.6 \mathrm{~ms} / 8^{\circ} / 15-20 \mathrm{~cm} / 1$ (TR/TE/flip angle/FOV/NEX), a $169 \times 169$ to $240 \times 240$ matrix, 1 -mm section thickness (0-mm gap), and an acquisition time of approximately 5 minutes; these scans were reconstructed in the axial plane at a section thickness of $3 \mathrm{~mm}$. For SE T1WI, the parameters were 353-734 $\mathrm{ms} / 10 \mathrm{~ms} / 14-20 \mathrm{~cm} / 1(\mathrm{TR} / \mathrm{TE} / \mathrm{FOV} / \mathrm{NEX})$, with a $168 \times 132$ to $265 \times 205$ matrix, axial 3-mm thickness (0.3-1.0 mm gap), and an acquisition time of about 5 minutes. We attempted to approximate and coregister the GE TIWI and SE TIWI to each other at the same thickness and level. Axial spin-echo T2WI, FLAIR, and DWI were also performed in each patient; the axial spin-echo T2WI and DWI acquisitions were performed after the intravenous administration of gadolinium-based contrast but prior to the postcontrast GE TIWI or SE TIWI acquisitions, to ensure a minimum delay of several minutes before the T1WI acquisitions were performed. The standard weightbased intravenous dose of gadolinium-based contrast was $0.1 \mathrm{~mL} / \mathrm{kg}$ of body weight $(0.1 \mathrm{mmol} / \mathrm{kg})$ of gadobutrol (Gadavist; Bayer Schering Pharma, Berlin, Germany).

\section{Imaging Interpretation}

Two staff neuroradiologists (A.M.M., B.K., each with $>10$ years of imaging experience) and 1 neuroradiology fellow (R.S.G., with 
Table 1: Demographics, TTI, dosages, and LMCE grades of the study patients

\begin{tabular}{llcc}
\hline \multicolumn{1}{c}{ Parameter } & Range & Mean & SD \\
\hline Age (yr) & $1.3-5.0$ & 3.1 & 1.4 \\
Weight (kg) & $5.7-24.6$ & 15.3 & 4.5 \\
Propofol dose (mcg/kg/min) & $73-303$ & 192 & 52 \\
Sedation duration (min) & $43-110$ & 66.6 & 13.8 \\
TTI GE TTWI (min) & $8.0-17.0$ & 12.6 & 2.2 \\
TTI SE TIWI (min) & $8.0-17.0$ & 11.0 & 2.1 \\
LMCE score on SE TIWI & $1.9-2.1$ & 2.0 & 0.8 \\
LMCE score on GE TIWI & $1.2-1.4$ & 1.2 & 0.8 \\
\hline
\end{tabular}

2 years of dedicated neuroradiology experience) independently graded the degree of LMCE as follows: grade 0, minimal thin vascular structures barely visible within the sulci; grade 1, thin vascular structures extending into the depths of the sulci; grade 2, smooth and slightly thickened LMCE; and grade 3, almost nodular, diffusely thickened LMCE or apparent involvement of adjacent parenchyma. The time between the commencement of the administration of intravenous contrast and the start of the postcontrast T1WI sequence was also recorded and was termed "time to imaging" (TTI).

\section{Statistical Analysis}

The interobserver variability was calculated regarding LMCE grades using the Cohen $\kappa$. The LMCE grade was correlated with the propofol dosage, duration of sedation, patient age, weight, and TTI using the Spearman correlation. A Mann-Whitney $U$ test was used to compare the grades of LMCE within the group $(n=6)$ who underwent both GE TIWI and SE TIWI. The significance threshold was set to $P<.05$.

\section{RESULTS}

Of 152 pediatric patients ( $1-5$ years of age) sedated by propofol for 3T MR imaging, 109 were excluded due to the lack of postcontrast T1WI $(n=96)$, the MR imaging being at 1.5T $(n=3)$, moderate-severe brain injury or congenital abnormalities, or several other factors, as listed under "Excluded Patients" within the organization chart of Fig 1. A total of 43 patients were ultimately included for MRI review; of these, 37 underwent postcontrast imaging with either solely gradient-echo T1WI $(n=20 / 43)$ or spin-echo T1WI ( $n=17 / 43)$; notably, 6 patients underwent both sequences $(n=6 / 43)$. Table 1 lists the mean patient age, weight, propofol dosage, sedation duration, and TTI for both sequences. While the postcontrast TTI range was similar between sequences, it was slightly greater on SE TIWI than on GE TIWI (mean, 12.6 versus 11.0 minutes), being significantly different $(P=.01)$.

As shown in Table 1, the range of LMCE grades of the reviewers was greater on SE TIWI (1.9-2.1) versus GE TIWI (1.2-1.4) and was significantly different $(P<.0001)$. Interobserver $\kappa$ between reviewers was strong for both GE TIWI $(\kappa=0.567-0.698$, $P<.0001)$ and SE TIWI $(\kappa=0.609-0.693, P<.0001)$. No patients had grade 0 LMCE on SE TIWI. Examples of the LMCE grades are provided in Figs 2-5.

Regarding the 6 patients who underwent both T1WI sequences, the mean LMCE grade on SE TIWI (1.83) was greater than that on GE TIWI (1.33) but was not significantly different $(P=.546)$. Examples of LMCE on both sequences in the same patient are shown in Figs 3 and 5.

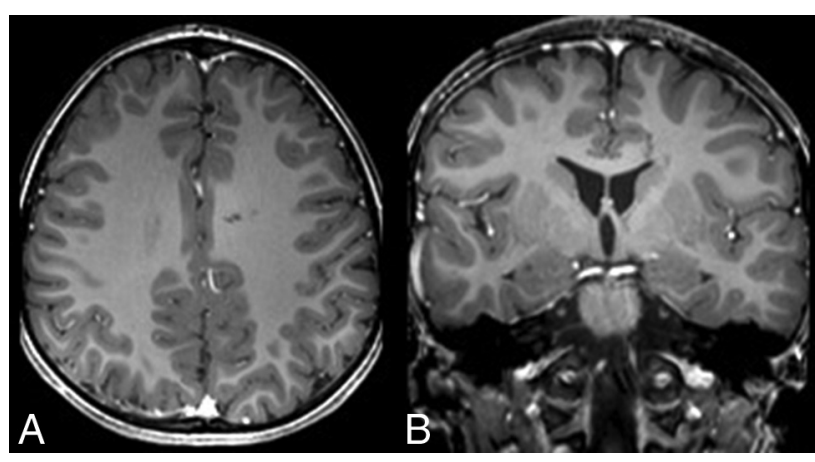

FIG 2. Grade 0 pseudo-LMCE in a 2-year-old girl post-trauma. Axial $(A)$ and coronal (B) GE TIWI shows only minimal vasculature within the sulci. This grade of enhancement was present only on GE TIWI in $13 \%-17 \%$, while no patients were graded as 0 on SE TIWI.

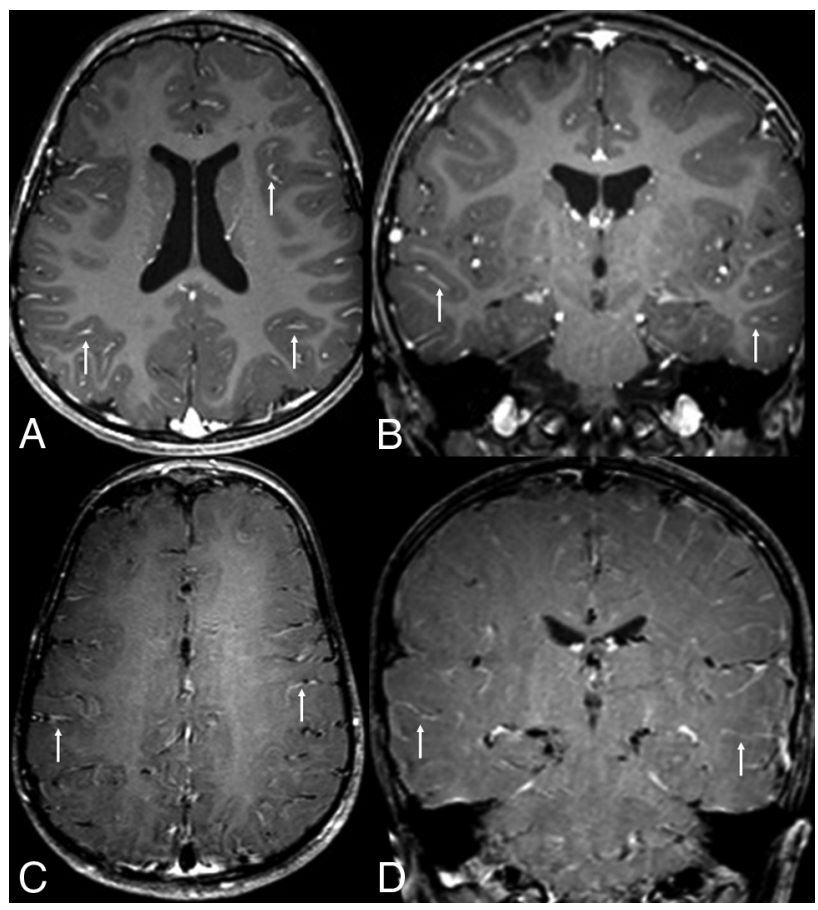

FIG 3. Grade 1 pseudo-LMCE on both sequences in a 3-year-old girl with seizures. Pseudo-LMCE appears as small vascular structures (arrows) within the depths of the sulci on GE TIWI axial $(A)$ and coronal $(B)$ images and on SE TIWI axial $(C)$ and coronal $(D)$ images. This grade was more frequent on GE TIWI (43\%-57\%) than on SE TIWI (25\%-30\%).

When we attempted to correlate various factors with the LMCE grade, there were significant, inverse, moderate correlations between patient weight and LMCE grade, as well as age and LMCE grade (each, $P<.05$; Table 2). Neither the propofol dose nor the sedation duration significantly correlated with the LMCE grade. The TTI did not correlate significantly with the grade of LMCE on GE TIWI, while on SE TIWI, there was a significant, moderate correlation between the LMCE grade and TTI with only 1 of the 3 observers (a staff neuroradiologist), but not the other 2 (Table 2).

\section{DISCUSSION}

Because MR imaging is noninvasive and does not use ionizing radiation, it is often a technique of choice for pediatric patients requiring neuroimaging. However, its potentially long imaging 


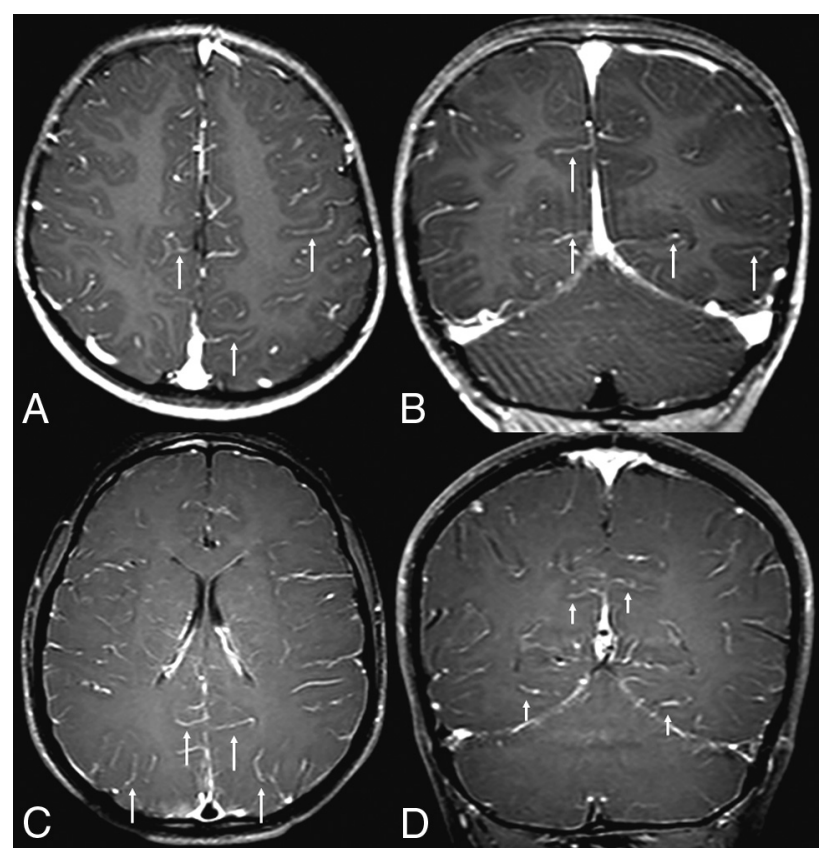

FIG 4. Examples of grade 2 pseudo-LMCE, demonstrated on both GE TIWI and SE TIWI in 2 different patients. In a 3-year-old girl with weakness, grade 2 pseudo-LMCE appears as smooth and slightly thickened enhancement (arrows) throughout the depths of the sulci on axial $(A)$ and coronal $(B)$ GE TIWI. In a 5-year-old boy with headaches, there is mildly thickened vasculature diffusely throughout the sulci (arrows) on axial (C) and coronal (D) SE TIWI. Note that grade 2 enhancement was slightly more frequent on SE TIWI (35\%-45\%) than on GE TIWI $(22 \%-35 \%)$.

time often requires sedation in pediatric patients younger than 8 years of age. Thus, propofol is a lipid emulsion agent that is commonly the preferred anesthetic for children younger than 1 year of age who require intravenous sedation, due to its rapid onset, short duration, and infrequent side effects. ${ }^{7-9}$ Because the presence of truly abnormal LMCE would be of concern in children, this study set out to determine whether pseudo-LMCE is a sequence-dependent (SE TIWI versus GE TIWI), TTI-dependent (time to postcontrast T1WI), propofol dosage-dependent phenomenon, or whether it is related to demographics such as age and weight. Ultimately, it was found that overall, the degree of apparent LMCE is significantly greater on SE TIWI compared with GE TIWI and that the only factors that correlated (inversely) with the degree of LMCE were patient weight and age. Hence, the type of T1WI sequence and patient size may be factors to consider when a pattern of apparent LMCE (so-called pseudo-LMCE) is identified, to distinguish this phenomenon from true meningeal abnormalities. Because this study focused solely on children between 1 and 5 years of age, future studies would be necessary to evaluate whether this phenomenon also occurs to some degree in older children and juveniles.

The mechanism of how this pattern of pseudo-LMCE occurs is not yet known, but there are several plausible explanations. The various determinants of cerebral blood flow are the patient's age, cerebral metabolic rate for oxygen, cerebral perfusion pressure, arterial oxygen, and carbon dioxide tensions. First, children under propofol sedation breathe spontaneously, but propofol causes a decrease in the tidal volume with a maintained respiratory rate

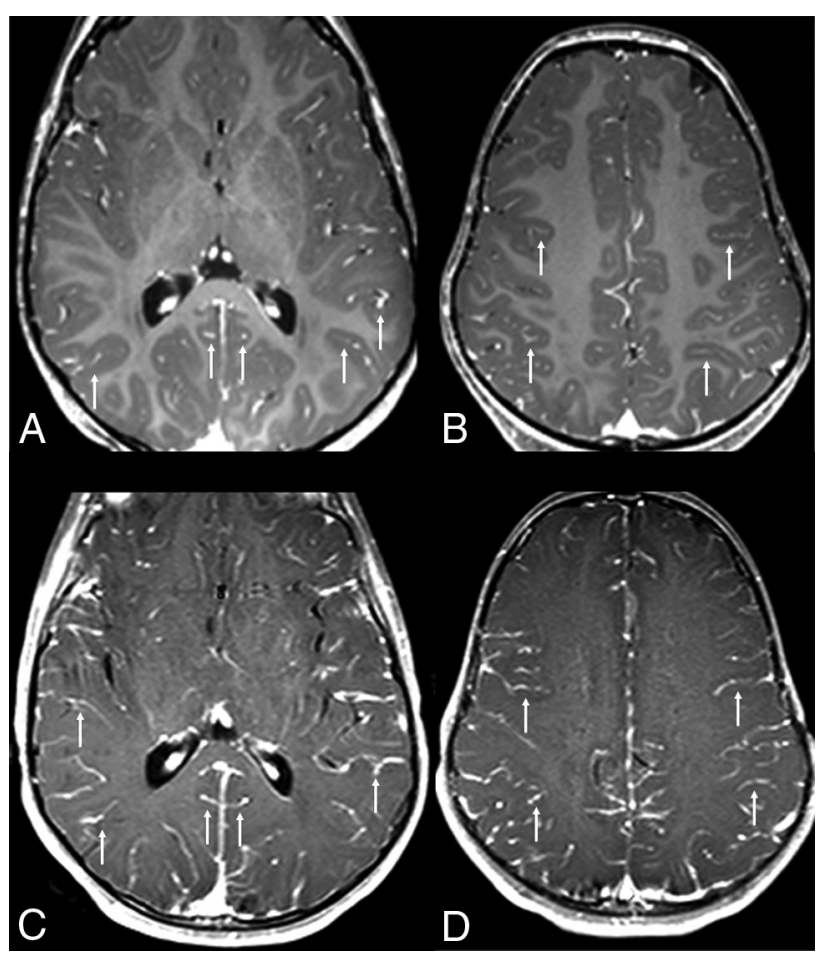

FIG 5. Discrepancy of the LMCE grade between sequences: grade 3 pseudo-LMCE on SE TIWI versus grade 2 on GE TIWI in a 3-year-old boy with fever. $A$ and $B$, Axial GE TIWI depicts irregular enhancement (arrows) throughout many of the sulci, being slightly thickened, consistent with grade 2 pseudo-LMCE. $C$ and D, Axial SE TIWI in the same patient demonstrates thicker pseudo-LMCE (arrows), appearing nearly nodular or parenchymal in some locations. This case demonstrates how such pseudo-LMCE is typically more prominent on SE TWI because grade 3 LMCE was much more frequent on SE TIWI $(30 \%-35 \%)$ than on GE TIWI (8\%-13\%).

and mild reduction in the partial pressure of oxygen in the blood, as well as a mild reduction in the fraction of inspired oxygen. By this phenomenon, one likely mechanism of pseudo-LMCE may be cerebral vasodilation secondary to an increase in the partial pressure of carbon dioxide (due to smooth-muscle relaxation); this increase in the partial pressure of carbon dioxide is likely due to the lack of "breathing off" $\mathrm{CO}_{2} \cdot{ }^{9}{ }^{910}$ Another factor could be that the leptomeninges may be more reactive or sensitive (in a sense "immature") in children compared with adults, an effect perhaps amplified by intravenous sedation. Additionally, because propofol sedation may affect respiration, studies have shown that the end-tidal volume of $\mathrm{CO}_{2}$ has an inverse relationship with the degree of venous contrast, which could also contribute to LMCE. ${ }^{11}$

Hence, on the basis of reviewing the images within this study as well as the authors' experience, the authors opine that pseudoLMCE on cerebral postcontrast T1WI often represents prominent venous vasculature of the subarachnoid space in younger children, being anecdotally described previously as less common in older children and adults; because older ages were not included in this study, this should be proved by a prospective study comparing age groups. ${ }^{12}$ However, the finding in the current study of a significant, inverse correlation between patient weight and the grade of pseudo-LMCE suggests that smaller and younger patients have more vasoreactivity, perhaps because their vasculature is not as mature. This theory may be supported by a study by 
Table 2: Correlation coefficients and $\boldsymbol{P}$ values for LMCE versus other factors ${ }^{\mathrm{a}}$

\begin{tabular}{lccccccc}
\hline Correlation & $\begin{array}{c}\text { TTI Overall } \\
\text { (SE and GE TIWI) }\end{array}$ & TTI SE TIWI Only & TTI GE TIWI Only & Weight $(\mathbf{k g})$ & Age (yr) & $\begin{array}{c}\text { Dose/Weight } \\
\text { (mg/kg) }\end{array}$ & $\begin{array}{c}\text { Duration of } \\
\text { Sedation }(\mathbf{m i n})\end{array}$ \\
\hline LMCE $(\rho)$ & -0.232 to -0.302 & -0.358 to -.475 & $0.016-0.190$ & -0.366 to -0.418 & -0.315 to -0.418 & $0.103-0.210$ & $0.023-0.147$ \\
$P$ value & $.051-.130$ & $.036^{\mathrm{b}}-.122^{\mathrm{c}}$ & $.371-.940$ & $.003-.011^{\mathrm{b}}$ & $.004-.032^{\mathrm{b}}$ & $.151-.484$ & $.318-.875$ \\
\hline
\end{tabular}

${ }^{a}$ Ranges provided are per the 3 reviewers.

${ }^{\mathrm{b}} P$ values $<.05$.

${ }^{c}$ Only 1 reviewer (a staff neuroradiologist) had a significant correlation between TTI on SE TIWI and degree of LMCE $(\rho=-0.475, P=.036)$

Harreld et al, ${ }^{4}$ which noted that in propofol-sedated children, the usual age-related decreases in CBF were reversed and increases in $\mathrm{CBF}$ and $\mathrm{CBV}$ were weight-dependent.

Unfortunately, exuberant pseudo-LMCE in children may simulate serious disorders that have implications for diagnosis therapy, such as leading to an unnecessary lumbar puncture to exclude meningitis. A radiologist should use other available imaging sequences to exclude true leptomeningeal abnormalities before the patient leaves the MR imaging scanner. The authors commonly experience the scenario in which a child with an unrelated diagnosis (eg, developmental delay, autism, and so forth) is imaged during sedation with propofol, in which the presence of pseudo-LMCE is spurious and varies with the postcontrast T1WI sequence used and the findings of other tests such as a resultant lumbar puncture, serum culture, and so forth are negative. This phenomenon being more common and greater in degree on SE TIWI versus GE TIWI is thought to be related to GE TIWI having a longer TR and lower contrast-to-noise ratio than SE TIWI; these features have been confirmed by studies noting that GE TIWI has a lower lesion detectability and visibility of contrast enhancement for a similar slice thickness. ${ }^{13-15}$ While there was a small difference in slice gap between the 2 sequences in this study, the slice thickness and acquisition plane were coregistered between the 2 sequences, so this small gap is unlikely to account for the difference in the degree of LMCE.

Intravenous contrast is not required in most pediatric brain MR imaging examinations, and gadolinium-based contrast should be avoided when unnecessary due to the possibility of deposition within particular brain structures, especially with repeat administrations in children. ${ }^{16-20}$ While this study did use a macrocyclic agent (the class of agents least likely to result in brain deposition), the use of most gadolinium-based agents is off-label for most gadolinium based intravenous contrast agents in the infantile population but is considered a standard of care in various clinical scenarios. ${ }^{16-18}$ For example, particular known or suspected pathologies that may require either gadolinium-based contrast for diagnosis or follow-up or to exclude related pathology including infectious disorders (eg, abscess, empyema, or meningoencephalitis), neoplasms, syndromic disorders (eg, phakomatoses), vascular malformations, or vasculitis, to name a few. Hence, while stewardship is critical to lessen the use of gadolinium-based contrast, there will continue to be subsets of patients that necessitate such contrast in the foreseeable future, and an awareness of this appearance of pseudo-LMCE may help prevent a misdiagnosis of leptomeningeal disease in children.

This study has several limitations, including its retrospective nature and the relatively small sample size of groups that underwent both T1WI sequences. The role of supplemental oxygen during sedation was not accounted for, which may also affect cerebral hemodynamics and alter subarachnoid signal intensity on other sequences, such as previously noted on FLAIR. ${ }^{21}$ In this regard, the authors found it difficult to obtain an accurate tabulation of the exact fractionation of oxygen and the length of time administered while the patient was under sedation, although the electronic record did note that there was titration of the supplemental oxygen in some patients. Thus, it is recommended that future studies prospectively tabulate the oxygen fraction accurately. Another potential limitation is that there was a small but significant difference between the TTI of both SE TIWI (12.6 minutes) and GE TIWI (11.0 minutes), which might create a bias toward having a greater LMCE score on SE TIWI; however, because no significant association was noted between the degree of LMCE and TTI, such bias (if present) was unlikely to affect the LMCE grade between sequences. Another limitation was that several factors such as CSF protein, fraction of inspired oxygen, end-tidal $\mathrm{CO}_{2}$, and leakage of propofol across the BBB were not evaluated in this study. These factors, previously implicated on T2WI and FLAIR imaging, could be assessed with respect to T1WI in a future study. ${ }^{21,22}$

\section{CONCLUSIONS}

The phenomenon of apparent LMCE, termed pseudo-LMCE herein, is relatively common on postcontrast T1-weighted MR imaging of younger children sedated by intravenous propofol and should not be mistaken for disease. This effect occurs more commonly and to a greater degree on SE TIWI compared with GE TIWI and inversely correlates with age and weight. The presence of this finding may relate to the immaturity of younger children's vasculature but needs to be studied further.

Disclosures: Alexander M. McKinney—UNRELATED: Board Membership: VEEV Inc Informatics, Comments: owner, Informatics Solutions.

\section{REFERENCES}

1. Smirniotopoulos JG, Murphy FM, Rushing EJ, et al. Patterns of contrast enhancement in the brain and meninges. Radiographics 2007; 27:525-51 CrossRef Medline

2. McKinstry CS, Worthington BS, Niendorf HP, et al. Demonstration of meningeal contrast enhancement on magnetic resonance imaging. Acta Radiol Suppl 1986;369:564-67 Medline

3. Kaisti KK, Långsjö JW, Aalto S, et al. Effects of sevoflurane, propofol, and adjunct nitrous oxide on regional cerebral blood flow, oxygen consumption, and blood volume in humans. Anesthesiology 2003; 99:603-13 CrossRef Medline

4. Harreld JH, Helton KJ, Kaddoum RN, et al. The effects of propofol on cerebral perfusion MRI in children. Neuroradiology 2013;55: 1049-56 CrossRef Medline

5. Klein KU, Fukui K, Schramm P, et al. Human cerebral microcirculation and oxygen saturation during propofol-induced reduction of bispectral index. Br J Anaesth 2011;107:735-41 CrossRef Medline 6. Gragasin FS, Davidge ST. The effects of propofol on vascular func-

AJNR Am J Neuroradiol 39:1739-44 Sep 2018 www.ajnr.org

1743 
tion in mesenteric arteries of the aging rat. Am J Physiol Heart Circ Physiol 2009;297:H466-74 CrossRef Medline

7. Machata AM, Willschke H, Kabon B, et al. Propofol-based sedation regimen for infants and children undergoing ambulatory magnetic resonance imaging. Br J Anaesth 2008;101:239-43 CrossRef Medline

8. Martin LD, Pasternak LR, Pudimat MA. Total intravenous anesthesia with propofol in pediatric patients outside the operating room. Anesth Analg 1992;74:609-12 Medline

9. Szabó EZ, Luginbuehl I, Bissonnette B. Impact of anesthetic agents on cerebrovascular physiology in children. Pediatr Anesth 2009;19: 108-18 CrossRef Medline

10. Remsen LG, Pagel MA, McCormick CI, et al. The influence of anesthetic choice, $\mathrm{PaCO} 2$, and other factors on osmotic blood-brain barrier disruption in rats with brain tumor xenografts. Anesth Analg 1999;88:559-67 Medline

11. Kwong KK, Wanke I, Donahue KM, et al. EPI imaging of global increase of brain MR signal with breath-hold preceded by breathing O2. Magn Reson Med 1995;33:448-52 CrossRef Medline

12. McKinney AM. Atlas of Normal Imaging Variations of the Brain, Skull, and Craniocervical Vasculature. New York: Springer-Verlag; 2017: 413-26; chap 18

13. Komada T, Naganawa S, Ogawa $\mathrm{H}$, et al. Contrast-enhanced MR imaging of metastatic brain tumor at 3 Tesla: utility of $\mathrm{T}(1)$ weighted SPACE compared with $2 \mathrm{D}$ spin echo and $3 \mathrm{D}$ gradient echo sequence. Magn Reson Med Sci 2008;7:13-21 CrossRef Medline

14. Chappell PM, Pelc NJ, Foo TK, et al. Comparison of lesion enhancement on spin-echo and gradient-echo images. AJNR Am J Neuroradiol 1994;15:37-44 Medline

15. Mugler JP 3rd, Brookeman JR. Theoretical analysis of gadopentetate dimeglumine enhancement in T1-weighted imaging of the brain: comparison of two-dimensional spin-echo and three-dimensional gradient-echo sequences. J Magn Reson Imaging 1993;3:761-69 CrossRef Medline

16. Saunders DE, Thompson C, Gunny R, et al. Magnetic resonance imaging protocols for paediatric neuroradiology. Pediatr Radiol 2007;37:789-97 CrossRef Medline

17. American College of Radiology. ACR Appropriateness Criteria. http://www.acr.org/Quality-Safety/Appropriateness-Criteria. Accessed May 1, 2018

18. Soares BP, Lequin MH, Huisman TA. Safety of contrast material use in children. Magn Reson Imaging Clin N Am 2017;25:779-85 CrossRef Medline

19. Roberts DR, Chatterjee AR, Yazdani M, et al. Pediatric patients demonstrate progressive T1-weighted hyperintensity in the dentate nucleus following multiple doses of gadolinium-based contrast agent. AJNR Am J Neuroradiol 2016;37:2340-47 CrossRef Medline

20. Ryu YJ, Choi YH, Cheon JE, et al. Pediatric brain: gadolinium deposition in dentate nucleus and globus pallidus on unenhanced T1weighted images is dependent on the type of contrast agent. Invest Radiol 2018;53:246-55 CrossRef Medline

21. Frigon C, Shaw DW, Heckbert SR, et al. Supplemental oxygen causes increased signal intensity in subarachnoid cerebrospinal fluid on brain FLAIR MR images obtained in children during general anesthesia. Radiology 2004;233:51-55 CrossRef Medline

22. Filippi CG, Ulug AM, Lin D, et al. Hyperintense signal abnormality in subarachnoid spaces and basal cisterns on MR images of children anesthetized with propofol: new fluid-attenuated inversion recovery finding. AJNR Am J Neuroradiol 2001;22:394-99 Medline 